\title{
Fuzzy Morphological Polynomial Image Representation
}

\author{
Chin-Pan Huang ${ }^{1}$ and Luis F. Chaparro (EURASIP Member) ${ }^{2}$ \\ ${ }^{1}$ Department of Computer and Communication Engineering, Ming Chuan University, Taoyuan 333, Taiwan \\ ${ }^{2}$ Department of Electrical and Computer Engineering, University of Pittsburgh, Pittsburgh, PA 15261, USA
}

Correspondence should be addressed to Chin-Pan Huang, hcptw@mail.mcu.edu.tw

Received 8 January 2010; Accepted 7 May 2010

Academic Editor: Srdjan Stankovic

Copyright (C) 2010 C.-P. Huang and L. F. Chaparro. This is an open access article distributed under the Creative Commons Attribution License, which permits unrestricted use, distribution, and reproduction in any medium, provided the original work is properly cited.

\begin{abstract}
A novel signal representation using fuzzy mathematical morphology is developed. We take advantage of the optimum fuzzy fitting and the efficient implementation of morphological operators to extract geometric information from signals. The new representation provides results analogous to those given by the polynomial transform. Geometrical decomposition of a signal is achieved by windowing and applying sequentially fuzzy morphological opening with structuring functions. The resulting representation is made to resemble an orthogonal expansion by constraining the results of opening to equate adapted structuring functions. Properties of the geometric decomposition are considered and used to calculate the adaptation parameters. Our procedure provides an efficient and flexible representation which can be efficiently implemented in parallel. The application of the representation is illustrated in data compression and fractal dimension estimation temporal signals and images.
\end{abstract}

\section{Introduction}

Signal representation is an area of great interest in the signal and image processing. Many representation techniques currently available are well developed and offer satisfactory performance in many applications [1-6]. However, in general, drawbacks of these techniques include intensive computation, sequential implementation, and disregard of geometrical information present in signals. In this paper, we propose fuzzy mathematical morphology [7] to represent one- and two-dimensional signals. Fuzzy morphological operators, similar to morphological operator, are nonlinear but well suited for efficient implementation in parallel. Furthermore, they allow to extract geometrical information in signals by appropriate transformations.

Among recently introduced representation techniques, Martens $[5,6]$ proposes a linear combination of polynomial to represent signals. Although the method achieves high performance in data compression, it has high computational complexity and a sequential implementation. Pitas and Venetsanopaulos [8-10] propose a morphological signal decomposition method to decompose a signal into a set of morphologically simple function. Song and Delp [11] use multiple structuring functions instead of a single function to enhance the performance of morphological filters. In developing the Fuzzy Morphological Polynomial (FMP) representation [12], we take the advantage of polynomial transform idea $[5,6]$, the morphological decomposition recursive procedures $[8-10]$ and using multiple structuring functions [11] overcoming some of the problems mentioned before.

Binary morphology, as developed in [13], is based on the concept of fitting a structuring element to the signal. Its extension to multilevel morphology was achieved by treating the space underneath the signal ("umbra") as a binary signal. Recently, Shinha and Dougherty [7] propose an alternate mathematical morphology based on fuzzy set theory. The morphological operations are modeled on a "fuzzy" notion of fitting, the umbra concept is not required and as such binary morphology becomes a special case. The fuzzy fitting yields one or zero for crisp fitting, and between zero and unity for a partial fit. The closer to unity, the higher the degree of fit.

The rest of the paper is organized as follows. In Section 2, we briefly review fuzzy mathematical morphology. Then in Section 3, we develop the one-dimensional FMP representation based on a recursive geometric decomposition for a given signal membership function. The properties of our 
FMP representation are investigated to develop algorithms to compute the adaptive parameters. In Section 4, we extend our algorithm to two-dimensions. We use the tensor product of the two one-dimensional functions as fuzzy structuring functions. The order of the two-dimensional fuzzy structuring functions is explored and a rational solution is recommended. In Section 5, we present our experiment results of applying our algorithm to data compression and fractal dimension estimation for one- and two-dimensional signals, demonstrating the advantages of our algorithm. The conclusion is in Section 6. Some of the results in this paper were presented before in [12].

\section{Fuzzy Mathematical Morphology}

Recently, Shinha and Dougherty [7] proposed to consider fuzzy set theory [14] instead of the classical set theory to develop mathematical morphology. They have in fact, obtained a new approach that considers simultaneously binary and multilevel morphology. The concept of "umbra" is no longer needed to develop the multilevel case. Morphological operations are then developed on the "fuzzy" fitting so that for crisp sets the fitting still remains characterized as either 0 or 1 , but for fuzzy or noncrisp sets it is possible to have a fitting characterized by a value between 0 and 1 . The closer to unity, the better the fitting of the structuring element. As in the classical morphology, fuzzy morphology [7] also consists in transforming a fuzzy set into another. Such a transformation is performed by means of a fuzzy structuring set containing the desired geometric structure.

If we let $X$ be the universe of discourse and $x$ be its generic element, the difference between crisp and fuzzy sets is the characteristic function of a crisp set $C$ which is defined as $\mu_{C}$ : $X \rightarrow\{0,1\}$ while the membership function $\mu_{F}: X \rightarrow[0,1]$ of a fuzzy set $F$ is defined so that $\mu_{F}(x)$ denotes the degree to which $x$ belongs to the set $F$. Among the different operations on fuzzy sets [15], the following are important operations to be used later.

(a) Complement operation:

$$
\mu_{F c}(x)=1-\mu_{F}(x) .
$$

(b) Translation of a fuzzy set $F$ by a vector $v \in X$ :

$$
\mu_{\mathcal{T}(F ; v)}(x)=\mu_{F}(x-v) .
$$

(c) Reflection of a set F:

$$
\mu_{-F}(x)=\mu_{F}(-x)
$$

(d) Bold union of two sets $F$ and $G$ :

$$
\mu_{F \Delta G}(x)=\min \left[1, \mu_{F}(x)+\mu_{G}(x)\right] .
$$

(e) Bold intersection $F \nabla G$ :

$$
\mu_{F \nabla G}(x)=\max \left[0, \mu_{F}(x)+\mu_{G}(x)-1\right] .
$$

The degree of fitting of a set $A$ into a set $B$ is measured by an inclusion grade operator

$$
\begin{aligned}
\ell(A, B) & =\inf _{x \in \mathrm{X}} \mu_{A^{c} \Delta B}(x) \\
& =1+\min \left\{0, \inf _{x \in X}\left[\mu_{B}(x)-\mu_{A}(x)\right]\right\},
\end{aligned}
$$

where $\Delta$ is the bold union operator. According to the above index, the degree of subsethood of two crisp sets $A, B$ is either 0 or 1 , while for fuzzy sets $C$ and $D \ell(C, D) \in[0,1]$. Moreover, if $C \subseteq D$ then $\ell(C, D)=1$ and in general $0 \leq \ell(C, D) \leq 1$. Using such an index [7] has shown that the erosion operation can be defined, and from it the dilation, opening, and closing operators are obtained. In fact, if $f(n)$ is a multilevel and $k(n)$ is a structuring element with supports $F$ and $K$ and membership function $\mu_{f}(n)$ and $\mu_{k}(n)$, then we have

Erosion:

$$
\begin{aligned}
\mu_{f \Theta k}(n) & =\ell(\mathcal{T}(k ; n), f) \\
& =\min _{i \in K}\left\{\min \left[1,1-\mu_{k}(i)+\mu_{f}(n+i)\right]\right\},
\end{aligned}
$$

Dilation:

$$
\begin{aligned}
\mu_{f \oplus k}(n) & =\mu_{\left(f^{c} \Theta-k\right)^{c}}(n) \\
& =\max _{i \in K}\left\{\max \left[0, \mu_{k}(i)+\mu_{f}(n-i)-1\right]\right\},
\end{aligned}
$$

Opening:

$$
\mu_{f \circ k}(n)=\mu_{(f \Theta k) \oplus k}(n)
$$

Closing:

$$
\mu_{f \bullet k}(n)=\mu_{(f \oplus k) \Theta k}(n)
$$

2.1. Fuzzification. To apply the above fuzzy morphological operators, the multilevel signal must be converted to its membership function. Thus, in case of an image, the membership function will determine the degree of belonging to categories ranging over different intensities. The membership function $\mu_{f}$ thus maps the image intensity range $R$ into $[0,1]$,

$$
\begin{aligned}
& \mu_{f}: R \longrightarrow[0,1] \\
& f(x, y) \longrightarrow[0,1],
\end{aligned}
$$

according to some criteria. Some of them are the Linear $(L)$ function

$$
\mu_{L}(f ; a, r)=\frac{f-a}{r-a}
$$

S-function

$$
\mu_{S}(f ; a, b, r)= \begin{cases}2\left(\frac{f-a}{r-a}\right)^{2}, & a \leq f \leq b, \\ 1-2\left(\frac{f-r}{r-a}\right)^{2}, & b \leq f \leq r,\end{cases}
$$




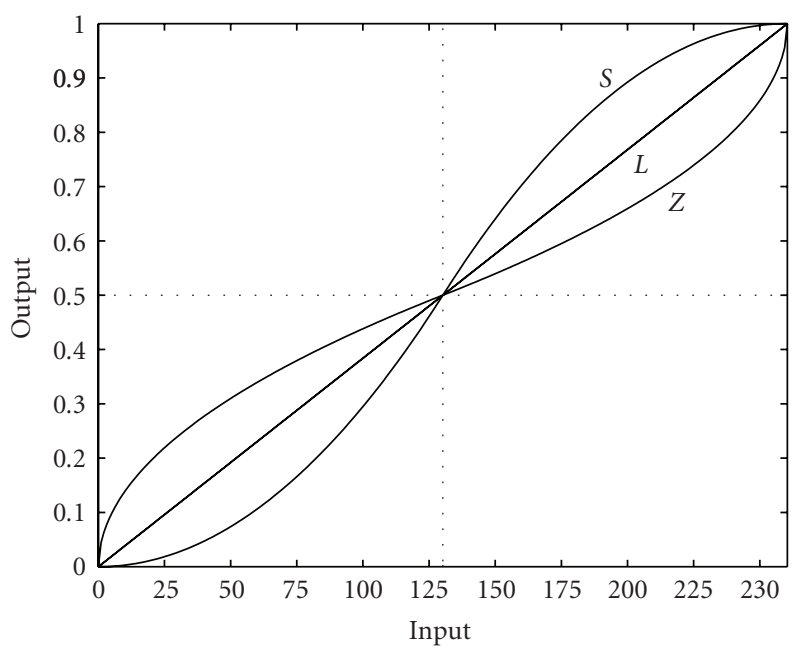

FIGURE 1: Fuzzification functions: $L, S, Z$ function.

and $\pi$-function $[16,17]$. In signal representation, the fuzzification needed to be single valued and as such we will not consider the $\pi$-function fuzzification. We propose a new fuzzification method, called $Z$-function fuzzification, which has the inverse effect of the $S$-function fuzzification. The $Z$ function is

$$
\mu_{Z}(f ; a, b, r)= \begin{cases}\sqrt{\frac{f-a}{2(r-a)},} & a \leq f \leq b, \\ 1-\sqrt{\frac{f-r}{2(a-r)}}, & b \leq f \leq r,\end{cases}
$$

where $\mu$ is the membership function and $f, a, b$, and $r$ are the grey level, and the minimum, middle, and maximum value of the image grey level. Notice that these three functions are all single valued and monotonically increasing in the analysis interval (minimum to maximum of the signal). The fuzzification functions for image is shown in Figure 1. Given that the fuzzification functions chosen are single valued, the defuzzification process is easily achieved by the inverse mapping.

The application of these fuzzification techniques in signal analysis varies. For instance, the $S$-function enhances the image contrast about the $b$ level, and as such it could be used for edge enhancement. On the other hand, the $Z$-function decreases the contrast or blurs the image about the $b$ level. The linear fuzzification does not alter the contrast, it simply normalizes the image to a range of $[0,1]$.

To illustrate the application of $S$-function in edge detection, consider the fuzzy morphological gradient (FMG)

$$
\mu_{d}(n)=\mu_{f \oplus k}(n)-\mu_{f \Theta k}(n)
$$

obtained as an extension of the classical morphological gradient first proposed by Serra [18] and Evans and Liu [19]. Applying the FMG to the real signal of an image in Figure 2(a), we obtain the lower figure where the peaks correspond to the edges. To enhance these edges, we consider the fuzzification of this result to achieve considerable enhancement of the edges as shown in Figure 2(b).

\section{Fuzzy Morphological Polynomial (FMP) Representation}

The FMP representation is analogous to the morphological polynomial transform [20] and the orthogonal polynomial representation [5]. Using fuzzy morphological opening we obtain a representation similar to a polynomial representation by means of a geometrical decomposition of the signal. One of the difficulties encountered in the process was the selection of the structuring functions, which can be either arbitrary or derived from the signal. In our case, we get them from a complete set of ordered real-valued orthogonal polynomials in $0 \leq n \leq N-1$. In the examples, we use the discrete Legendre orthogonal (DLO) polynomials [21]. It should be noted that the corresponding membership functions are not necessarily orthogonal. Let $\mu_{f}(n)$ be the membership function of a given signal, and $\left.\mu_{k_{i}}(n): 0 \leq i \leq N-1\right\}$ be one-dimensional fuzzy structuring functions, such that $0 \leq \mu_{k_{i}}(n) \leq 1$. Let $\left\{a_{i}\right\}$ be adaptive parameters used to make the fuzzy structuring function fit $\mu_{f}(n)$ closely. To consider all possibilities, the fuzzy structuring functions $\left\{\mu_{k_{i}}(n)\right\}$ are derived from a shifted and normalized set of orthogonal polynomials $\left\{\mu_{g_{i}}(n)\right\}$ and its complementary functions $\left\{\mu_{g_{i}^{c}}(n)\right\}$. Figure 3 illustrates the shifted and normalized functions $\left\{\mu_{g_{i}}(n)\right\}$ when we consider the discrete Legendre orthogonal polynomials for $N=5$.

The geometric decomposition of the given membership function $\mu_{f}(n)$ is obtained recursively as follows.

(i) Windowing with $W(n)$ :

$$
\mu_{z_{0}}^{v}(n)=\mu_{f}(n) \times W(n-v N) .
$$

(ii) Adaptive recursive approximation of $\mu_{z_{0}}(n)$ :

$$
\mu_{z_{i+1}}^{v}(n)=\mu_{z_{i}}^{v}(n)-\mu_{z_{i} \circ a_{i} k_{i}}^{v}(n)
$$

where $i=0,1, \ldots, N-1$ relates to the structuring functions $a_{i} \mu_{k_{i}}(n), v=0,1,2, \ldots$ refers to the window $W$, and $a_{i}$ are in $[0,1]$ adaptive parameter. Each window is processed similarly.

The term $a_{0} \mu_{k_{0}}(n)$ is very important in the above decomposition as it provides a coarse approximation to the signal membership function while $\left\{a_{i} \mu_{k_{i}}(n), i>0\right\}$ gives the fine information of $\mu_{z_{0}}^{v}(n)$. Applying (17) recursively we have

$$
\mu_{z_{0}}^{v}(n)=\sum_{i=0}^{N-1} \mu_{z_{i} \circ a_{i} k_{i}}^{v}(n)+\mu_{z_{N}}^{v}(n),
$$

where the last term corresponds to the residual or the part of the signal that cannot be well represented with $N$ function $\mu_{k_{i}}(n)$. We will show in the next section that the above representation can be considerably simplified by choosing values of $\left\{a_{i}\right\}$ such that

$$
\mu_{z_{i} \circ a_{i} k_{i}}^{v}(n)=a_{i} \mu_{k_{i}}(n)
$$

to convert (18) into

$$
\mu_{z_{0}}^{v}(n)=\sum_{i=0}^{N-1} a_{i} \mu_{k_{i}}(n)+\mu_{z_{N}}^{v}(n),
$$




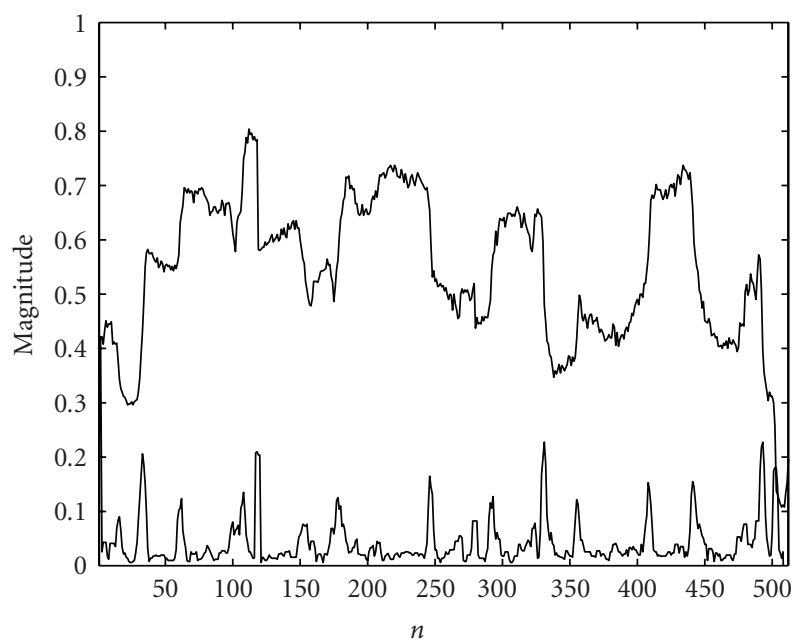

(a)

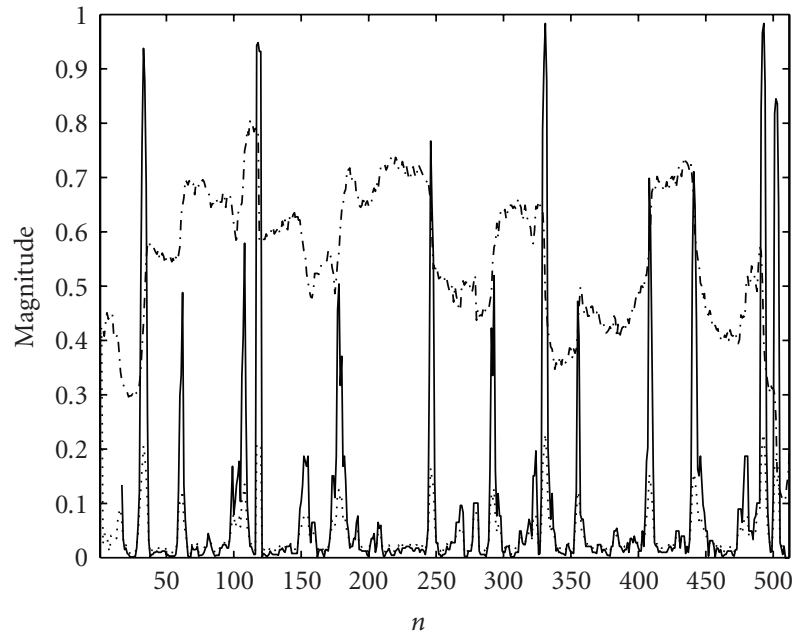

(b)

Figure 2: Effects of $S$-function on edge enhancement (a) Original signal and edge detected, (b) $S$-function enhanced version (solid edges) of (b).

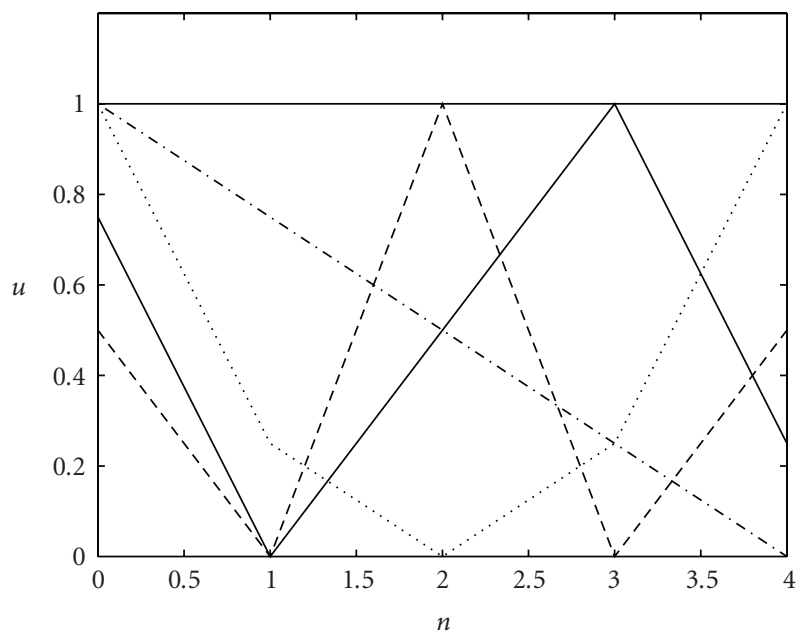

FIGURe 3: Fuzzy structuring functions using DLO polynomials for $N=5$.

representation analogous to a polynomial representation of the windowed signal.

Properties. The following propositions will give insight on how the FMP representation works and how to develop formulas to calculate the $\left\{a_{i}\right\}$ coefficients. Here, we work on a frame signal only, and thus the superscript $v$ can be omitted. Also, we use $\min _{\ell}, \max _{\ell}$ to stand for $\min _{0 \leq \ell \leq N-1}$ and $\max _{0 \leq \ell \leq N-1}$. In the following propositions, we assume $\mu_{z}(n), \mu_{k}(n)$ are both defined on $0 \leq n \leq N-1$, and $\left\{a_{i}\right\} \in[0,1]$ then

\section{Proposition 1. The opening}

$$
\mu_{z_{i} \circ a_{i} k_{i}}^{v}(n)=\max \left[0, a_{i} \mu_{k_{i}}(n)+\mu_{c}-1\right],
$$

where $\mu_{c}=1+\min \left\{0, \min _{\ell}\left[\mu_{z_{i}}(\ell)-a_{i} \mu_{k_{i}}(\ell)\right]\right\}$.
This proposition provides a simplification of the nonlinear opening and yield $\mu_{c}$, an index of the degree of fitting of the structuring function in the signal membership function.

Proposition 2. There exists an optimum $\left\{a_{i}\right\} \in[0,1]$ (denoted as $a_{i}^{*}$ ) such that $\mu_{z_{i} \circ a_{i}^{*} k_{i}}^{v}(n)=a_{i}^{*} \mu_{k_{i}}, 0 \leq n \leq N-1$, if and only if the following optimum condition is satisfied

$$
\min _{\ell}\left[\mu_{z_{i}}(\ell)-a_{i}^{*} \mu_{k_{i}}(\ell)\right]=0 .
$$

The value $a_{i}^{*}$ is calculated as

$$
a_{i}^{*}=\min _{\substack{0 \leq l \leq N-1 \\ \mu_{k_{i}}(l) \neq 0}}\left\{\frac{\mu_{z_{i}}(\ell)}{\mu_{k_{i}}(\ell)}\right\} .
$$

Let $J\left(a_{i}\right)=\min _{\ell}\left[\mu_{z_{i}}(\ell)-a_{i} \mu_{k_{i}}(\ell)\right]$ be the fitting cost function, and refer to

$$
J\left(a_{i}^{*}\right)=0
$$

as the optimum condition. There are two direct corollaries that follow from this proposition.

Corollary 1. If the optimum condition is met, then, there are the following.

(i) If $a_{i}>a_{i}^{*}$, then $J\left(a_{i}\right)<0,0 \leq n \leq N-1$,

(ii) If $a_{i}<a_{i}^{*}$, then $J\left(a_{i}\right)>0,0 \leq n \leq N-1$,

(iii) $a_{i}^{*}=\min _{n}\left[\mu_{z_{0}}(n)\right]$.

(iv) $0 \leq a_{i}^{*} \leq \max \left[\mu_{z_{i}}(n)\right] \leq 1,0 \leq i \leq N-1$.

Corollary 2. If the optimum condition is met for $\mu_{z_{0}^{c}}(n)$, then

$$
a_{0}^{* c}=\max _{n}\left[\mu_{z_{0}}(n)\right] .
$$

Proposition 2 gives a solution to the optimal fitting problem. In the next section, we develop a geometrically intuitive solution. 
Proposition 3. For $\mu_{z_{i}}(n), i=0,1, \ldots, N-1$ in (17), the following relation holds

(i) $0 \leq \mu_{z_{i+1}}(n) \leq \mu_{z_{i}}(n) \leq 1$,

(ii) $\min _{n}\left[\mu_{z_{i+1}}(n)\right]=0$.

This proposition shows that the residual at each decomposition step satisfies the membership function conditions, decreases, and at least in one point is zero.

Proposition 4. If the optimum condition is met for $a_{i}$ and $a_{i}^{\prime}$, then the only value of $a_{i}^{\prime}$ is zero to satisfy the following equations $\mu_{z_{i} \circ a_{i} k_{i}}(n)=a_{i} \mu_{k_{i}}(n), \mu_{z_{i}^{\prime} \circ a_{i}^{\prime} k_{i}}(n)=a_{i}^{\prime} \mu_{k_{i}}(n)$, for $0 \leq i \leq N-1$, where $\mu_{z_{i}^{\prime}}(n) \triangleq \mu_{z_{i}}(n)-a_{i} \mu_{k_{i}}(n)$.

This proposition establishes that once geometrical features are decomposed from an input membership function, opening with previously used fuzzy structuring function gives zero.

3.1. Implementation. The representation of $\mu_{f}(n)$ according to (20) requires the calculation of the adaptive coefficient $\left\{a_{i}\right\}$ and choosing the appropriate structuring functions. There are two possible methods to find the coefficients $\left\{a_{i}\right\}$, the first one is based on a geometrically intuitive argument, while the second uses Proposition 2 given before. Consider a given window, and for simplicity let us not indicate it in the equation.

\subsubsection{Calculation of $\left\{a_{i}\right\}$}

Iterative Method. Let $a_{i}^{q}$ be the $q$ th iteration of $a_{i}\left(a_{i}^{*}\right.$ as the optimum) when attempting to optimize the fitting cost function

$$
J\left(a_{i}\right)=\min _{0 \leq \ell \leq N-1}\left[\mu_{z_{i}}(\ell)-a_{i} \mu_{k_{i}}(\ell)\right],
$$

with respect to $a_{i}$, the optimum value $a_{i}^{*}$ is obtained when the cost is zero, and such that $\mu_{z_{i} \circ a_{i}^{*} k_{i}}(n)=a_{i}^{*} \mu_{k_{i}}(n)$.

According to (26) and Corollary 1, the following algorithm can be used to find the optimum value of $a_{i}$ :

$$
\begin{gathered}
a_{i}^{0}=\max \left[\mu_{z_{i}}(n)\right], \\
J\left(a_{i}^{q}\right)=\min _{0 \leq \ell \leq N-1}\left[\mu_{z_{i}}(\ell)-a_{i}^{q} \mu_{k_{i}}(\ell)\right], \\
a_{i}^{q+1}=a_{i}^{q}+J\left(a_{i}^{q}\right),
\end{gathered}
$$

where $q \geq 0$. That $J\left(a_{i}^{q}\right)$ converges toward zero when $q$ increases can be established. If we assume that $a_{i}^{q}>a_{i}^{*}$, then $J\left(a_{i}^{q}\right) \leq 0$ (Corollary 1$)$, and, thus

$$
\begin{aligned}
J\left(a_{i}^{q+1}\right) & =\min _{0 \leq \ell \leq N-1}\left[\mu_{z_{i}}(\ell)-\left(a_{i}^{q}+J\left(a_{i}^{q}\right)\right) \mu_{k_{i}}(\ell)\right] \\
& \geq \min _{0 \leq \ell \leq N-1}\left[\mu_{z_{i}}(\ell)-a_{i}^{q} \mu_{k_{i}}(\ell)\right]=J\left(a_{i}^{q}\right) .
\end{aligned}
$$

Direct Method. As in Proposition 2, we can compute $a_{i}^{*}$ using (23). This method gives the same $a_{i}^{*}$ as the iterative method, but in a faster way.
3.1.2. Choosing Structuring Functions. Given shifted and normalized orthogonal polynomials $\left\{\mu_{g_{i}}(n)\right\}$ and their complements $\left\{\mu_{g_{i}^{c}}(n)\right\}$, we need to determine which of these two should be used as the structuring functions $\left\{\mu_{k_{i}}(n)\right\}$ for the representation. This needs to be done due to the positivity condition on the adaptation coefficients $\left\{a_{i}\right\}$. We decide this by comparing the reconstruction error corresponding to the coefficients attached to each of these structuring functions.

Let the coefficients $\hat{a}_{i}$ and $\tilde{a}_{i}$ be the optimum values for $\mu_{g_{i}}(n)$ and $\mu_{g_{i}^{c}}(n)$, respectively. We want to choose the optimum value which gives us the smaller reconstruction error. Let the reconstruction error membership function corresponding to $\hat{a}_{i}$ and $\tilde{a}_{i}$ be, respectively,

$$
\begin{aligned}
& \mu_{e_{1}}(n)=\mu_{z_{i}}(n)-\hat{a}_{i} \mu_{g_{i}}(n), \\
& \mu_{e_{2}}(n)=\mu_{z_{i}}(n)-\tilde{a}_{i} \mu_{g_{i}^{c}}(n) .
\end{aligned}
$$

It can be easily shown that

$$
\alpha_{i} \sum_{n=0}^{N-1} \mu_{g_{i}}(n)=\sum_{n=0}^{N-1} \mu_{g_{i}^{c}}(n),
$$

where $\alpha_{i}$ is found to be

$$
\alpha_{i}=\frac{N}{\sum_{n=0}^{N-1} \mu_{g_{i}}(n)}-1
$$

(notice $\alpha_{0}=0$ and $1-\mu_{k_{0}}(n)=0$, so $i \geq 1$ ). If

$$
\hat{a}_{i} \leq \tilde{a}_{i} \alpha_{i}
$$

then we have that

$$
\sum_{n=0}^{N-1} \mu_{e_{1}}(n) \geq \sum_{n=0}^{N-1} \mu_{e_{2}}(n) .
$$

In that case, we then let $\alpha_{i}=\tilde{a}_{i}$ and $\mu_{k_{i}}(n)=1-\mu_{g_{i}}(n)$. Otherwise, we choose $\alpha_{i}=\hat{a}_{i}$ and $\mu_{k_{i}}(n)=\mu_{g_{i}}(n)$.

\section{Two-Dimensional Fuzzy Morphological Polynomial Representation}

The FMP representation can be easily generalized to two dimensions. Let $\mu_{f}(m, n)$ be the given signal membership function and, $\mu_{k_{\varnothing}}(m, n), 0 \leq \varnothing \leq M N-1$ be ordered twodimensional fuzzy structuring functions, based on orthogonal polynomials on $0 \leq m \leq \mathrm{M}-1,0 \leq n \leq N-1$. The geometrical decomposition algorithm becomes

$$
\begin{gathered}
\mu_{z_{0}}^{u, v}(m, n)=\mu_{f}(m, n) \times W(m-u M, n-v N), \\
\mu_{z_{\varnothing+1}}^{u, v}(m, n)=\mu_{z_{\varnothing}}^{u, v}(m, n)-\mu_{z_{\varnothing} \circ a_{\varnothing} \mu_{k_{\varnothing}}}^{u, v}(m, n),
\end{gathered}
$$

where $\varnothing=0,1, \ldots, M N-1$ is an index related to the adaptive structuring function $a_{\varnothing} \mu_{k_{\varnothing}}(m, n)$; and $u, v$ are indices of the block being considered. The above procedure is repeated until the residual is insignificant or $\varnothing=M N-1$. Each 
block is decomposed similarly. As in (18)-(20), our twodimensional FMP representation for a frame signal is

$$
\mu_{z_{0}}^{u, v}(m, n)=\sum_{\varnothing=0}^{M N-1} a_{\varnothing} \mu_{k_{\varnothing}}^{u, v}(m, n)+\mu_{z_{M N}}^{u, v}(m, n)
$$

Notice that the $\varnothing$, abbreviation of $\varnothing(i, j)$, is an ordering function used for the two-dimensional structuring functions. The properties of one-dimensioned FMP can be extended to two dimensions easily, thus, we omit the derivation here. As in the one dimension, the optimum condition in the two dimensions is

$$
\min _{s, t}\left[\mu_{z \varnothing}(s, t)-a_{\varnothing}^{*} \mu_{k_{\varnothing}}(s, t)\right]=0,
$$

where $a_{\varnothing}^{*}$ is an optimum value to satisfy this equation.

It is understood from the previous section that the one-dimensional algorithm can be extended to twodimensional provided the generation and ordering of the two-dimensional structuring functions are determined. Separable and nonseparable bivariate orthogonal polynomials may be used to generate two-dimensional structuring functions. Consider the separable structuring functions

$$
\mu_{k_{i, j}}(m, n)=\mu_{k_{i}}(m) \mu_{k_{j}}(n)
$$

obtained from the one-dimensional structuring functions. Figure 4 shows an example of two-dimensional separable structuring functions with size of $5 \times 5$.

An inherent problem in two dimensions is the ordering of the structuring functions, which in the one-dimensional case occurs naturally. Our approach first investigates the structuring function properties and establishs the possible guides to the order of the two-dimensional structuring functions and then comes out a procedure to get the solution.

Proposition 5. If one multiplies two normalized one variate structuring functions, derived from the discrete Legendre orthogonal polynomials, $\mu_{k_{i}}(m)$ of size $M$ and $\mu_{k_{j}}(n)$ of size $N$ as two-dimensional structuring function, that is, $\mu_{k_{i, j}}(m, n)=$ $\mu_{k_{i}}(m) \mu_{k_{j}}(n)$ with dimension of $M \times N$, then one can have the following properties:

(i) $\mu_{k_{0, j}}(m, n) \geq \mu_{k_{i, j}}(m, n)$ for all $i, j, m, n$.

(ii) $\mu_{k_{i, 0}}(m, n) \geq \mu_{k_{i, j}}(m, n)$ for all $i, j, m, n$.

(iii) $\mu_{k_{0,0}}(m, n) \geq \mu_{k_{i, j}}(m, n)$ for all $i, j, m, n$.

This proposition gives properties of the separable two-dimensional structuring functions based on onedimensional DLO polynomials.

Proposition 6. If optimum condition is met for $a_{\varnothing(i, j)}^{*}$ and $a_{\varnothing(s, t)}^{*}$ and $\mu_{k_{\varnothing(i, j)}}(m, n) \leq \mu_{k_{\varnothing(s, t)}}(m, n)$ for a pair $(i, j),(s, t)$ and for all $m, n$, then the only value of $a_{\varnothing(s, t)}^{*}$ is zero to satisfy the following equations:

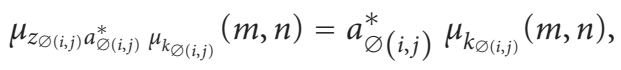

$$
\begin{aligned}
& \mu_{z \varnothing(s, t)} a_{\varnothing(s, t)}^{*} \mu_{k_{\varnothing(s, t)}}(m, n)=a_{\varnothing(s, t)}^{*} \mu_{k_{\varnothing(s, t)}}(m, n),
\end{aligned}
$$

where $\mu_{z_{\varnothing(s, t)}}(m, n) \triangleq \mu_{z_{\varnothing(i, j)}}(m, n)-a_{\varnothing(i, j)}^{*} \mu_{k_{\varnothing(i, j)}}(m, n)-$ $\epsilon(m, n)$, with $0 \leq \epsilon(m, n) \leq 1$, for all $m, n$ and arbitrary such that $0 \leq \mu_{z_{\varnothing(s, t)}}(m, n) \leq 1$.

This proposition gives guides of the structuring function order. If the structuring function has higher amplitude values than the other one at every point, then the structuring function must have a lower order otherwise the decomposition gets zero.

By observation, the factor in addition to the amplitude which affect the natural order of the one-dimensional structuring function is its complexity. Based on the properties and the observation, we come up with our rationale solution. We first define the amplitude and complexity index to quantitatively measure the structuring function amplitude and complexity characteristic.

Definition 1. An amplitude index (AI) of a geometrical structuring function $\mu_{k_{i, j}}(m, n)$ of size $M \times N$ is defined by the summation of amplitude at every pixel as

$$
\mathrm{AI}(i, j)=\sum_{n=0}^{N-1} \sum_{m=0}^{M-1} \mu_{k_{i, j}}(m, n) .
$$

Definition 2. A complexity index (CI) of a geometrical structuring function $\mu_{k_{i, j}}(m, n)$ of size $M \times N$ is defined by the distance between the adjacent pixel in the horizontal, vertical, and diagonal directions as

$$
\begin{aligned}
\mathrm{CI}(i, j)= & \sum_{m=0}^{M-1} \sum_{n=0}^{N-1}\left|\mu_{k_{i, j}}(m, n)-\mu_{k_{i, j}}(m, n+1)\right|^{2} \\
& +\sum_{m=0}^{M-2} \sum_{n=0}^{N-1}\left|\mu_{k_{i, j}}(m, n)-\mu_{k_{i, j}}(m+1, n)\right|^{2} \\
& +\sum_{m=0}^{M-2} \sum_{n=0}^{N-2}\left|\mu_{k_{i, j}}(m, n)-\mu_{k_{i, j}}(m+1, n+1)\right|^{2} \\
& +\sum_{m=1}^{M-1} \sum_{n=0}^{N-2}\left|\mu_{k_{i, j}}(m, n)-\mu_{k_{i, j}}(m-1, n+1)\right|^{2} .
\end{aligned}
$$

Based on the CI definition, the membership function is the simplest (constant) only if $\mathrm{CI}(i, j)=0$; if two fuzzy structuring functions have the same geometrical structures then they have the same complexity index $\mathrm{CI}(i, j)$ value.

The effects of the amplitude and complexity index of the structuring function to order are that the smaller the amplitude index, the higher the order of the structuring function, and the greater the complexity index, the higher the order of that structuring function. We combine these definitions and their effects and come out, the structuring index (SI). 

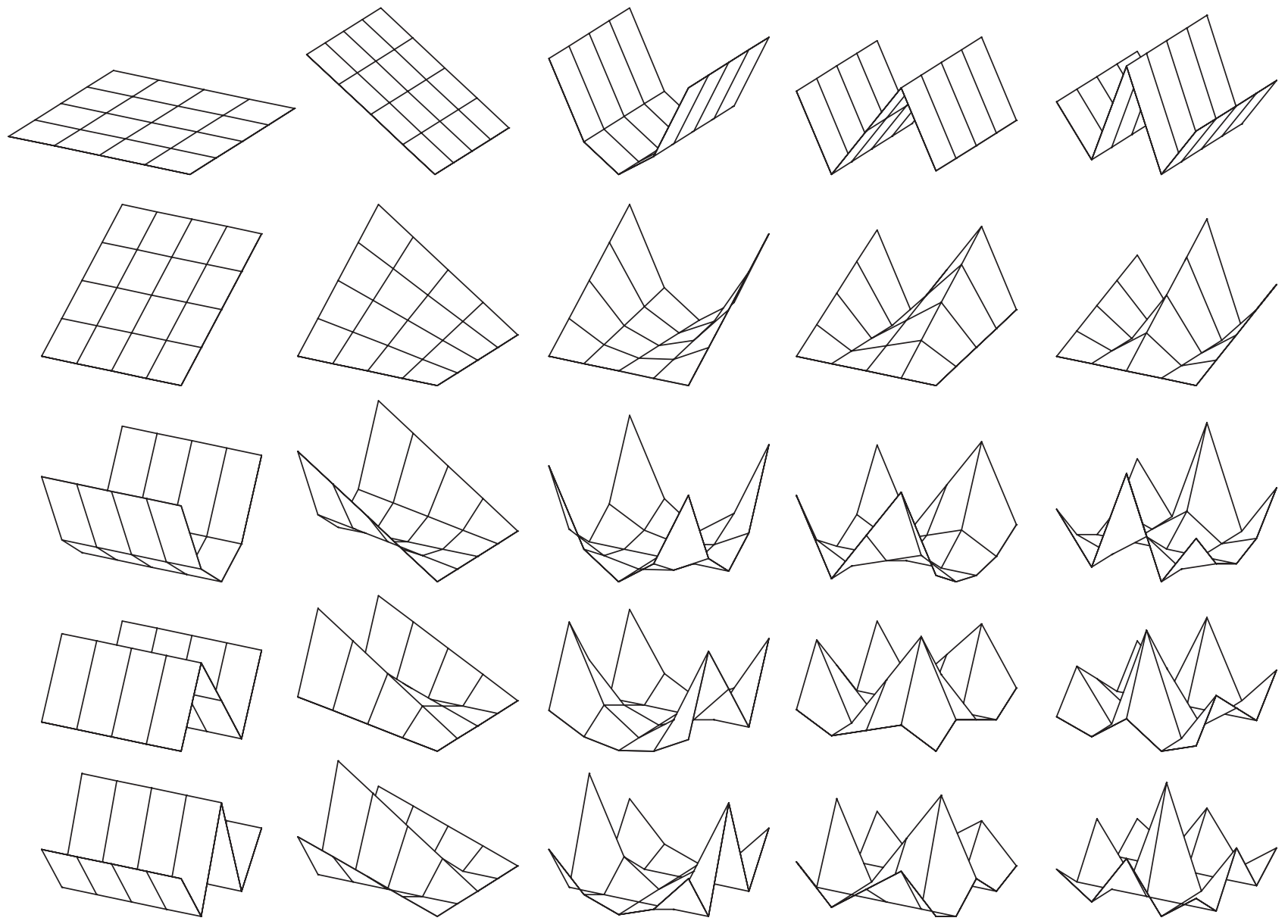

Figure 4: Two-dimensional separable structuring functions.

Definition 3. A structuring index (SI) of a structuring function $\mu_{k_{\mathrm{ij}}}(m, n)$ with amplitude index $\mathrm{AI}(i, j)$ and complexity index $\mathrm{CI}(i, j)$ is defined as

$$
\mathrm{SI}(i, j)=0.5\left[\mathcal{N}\left(\mathrm{AI}(i, j)^{-1}\right)+\mathcal{N}(\mathrm{CI}(i, j))\right],
$$

where 0.5 is average factor and $\mathcal{N}(\cdot)$ is a normalization operator over the structuring functions.

Notice that the value of SI is in $[0,1]$.

Based on $\operatorname{SI}(i, j)$, the order of the fuzzy structuring functions is

$$
\begin{aligned}
& \varnothing(i, j)<\varnothing(s, t), \\
& \text { iff } \operatorname{SI}(i, j)<\operatorname{SI}(s, t) \text {, } \\
& \text { or } \operatorname{SI}(i, j)=\operatorname{SI}(s, t) \text {, }
\end{aligned}
$$

We can get the order of the two-dimensional fuzzy structuring functions from one variate DLO polynomials. As an example, this will order $5 \times 5$ two-dimensional structuring functions as Table 1. Our ordering method, considering both the amplitude and intrinsic geometrical complexity of the
TABLE 1: Order of the structuring functions $\mu_{k_{i, j}}(m, n)$ of size $5 \times 5$.

\begin{tabular}{ccccccc}
\hline & & & $j$ & & \\
& & 0 & 1 & 2 & 3 & 4 \\
\hline \multirow{4}{*}{$i$} & 0 & $(0)$ & $(1)$ & $(4)$ & $(6)$ & $(16)$ \\
& 1 & $(2)$ & $(3)$ & $(8)$ & $(10)$ & $(8)$ \\
& 2 & $(5)$ & $(9)$ & $(12)$ & $(13)$ & $(20)$ \\
& 3 & $(7)$ & $(11)$ & $(14)$ & $(15)$ & $(22)$ \\
& 4 & $(17)$ & $(19)$ & $(21)$ & $(23)$ & $(24)$ \\
\hline
\end{tabular}

structuring function, is more reasonable than the commonly used close-neighbor ordering method [22], considering only the index of the structuring functions.

\section{Applications}

In this section, we show how the FMP representation is applied to data compression and fractal dimension estimation. We compare the data compression results with those using the discrete cosine transform (DCT) method [23], and the fractal dimension estimation results with those using morphological covering (MC) method [24] and differential box-counting (DBC) method [25]. 
TABLE 2: Signal to noise ratio $(\mathrm{dB})$ for one-dimensional FMP.

\begin{tabular}{lcccccccc}
\hline & \multicolumn{1}{c}{$L$} & \multicolumn{4}{c}{$Z$} & \multicolumn{3}{c}{$S$} \\
& \multicolumn{4}{c}{ window sizes } \\
\multicolumn{1}{c}{3} & 4 & 5 & 3 & 4 & 5 & 3 & 4 & 5 \\
\hline 125.53 & 23.43 & 21.84 & 25.53 & 23.43 & 21.84 & 25.53 & 23.43 & 21.84 \\
232.72 & 28.59 & 26.04 & 32.46 & 28.43 & 25.93 & 32.71 & 28.58 & 26.01 \\
334.60 & 29.57 & 27.09 & 33.37 & 29.16 & 26.65 & 33.74 & 29.17 & 26.16 \\
4 & 30.07 & 27.55 & & 29.67 & 27.20 & & 29.71 & 26.52 \\
5 & & 27.95 & & & 27.56 & & & 26.56 \\
\hline
\end{tabular}

5.1. Data Compression. The application of the FMP representation for data compression is shown in Figure 5. The signal $f(n)$ is fuzzified by $\mathcal{F}$ and then processed by the FMP decomposition to get the adaptive coefficients. The signal membership function is reconstructed and $\tilde{f}(n)$ is recovered by the defuzzifier $\mathscr{D}$. The block diagram for two dimensional signals is similar. The pepper image with size of $512 \times 512$ shown in Figure 6 is used as a test image.

In the first example, the one-dimensional FMP algorithm is used to process the test image horizontally. We consider different fuzzification methods, window lengths. The fuzzy structure functions are obtained from DLO polynomials as shown before. The performance of our representation is evaluated by the "peak-to-peak" signal to noise ratio (SNR $\mathrm{dB}$ ) and the entropy-based compression ratio (ECR). The entropy-based compression ratio (ECR) is defined as

$$
\mathrm{ECR}=\frac{\text { total l.c.of bits of compressed signal }}{\text { total l.c.of bits of original signal }}=\frac{\sum_{i=0}^{N-1} M_{i} l_{i}}{M_{T} l_{T}},
$$

where $N$ is the number of subblock signals, $M_{i}$ is the number of samples of the subblock $i, l_{i}$ is the bits/sample required to code subblock $i, l_{T}$ is the bits/sample required for the original signal, $M_{T}$ is the total number of samples of the original signal. The average bits/sample $l_{i}$ required to code a subblock signal is defined by entropy as

$$
l_{i}=-\sum_{j=0}^{G-1} p_{j} \log _{2} p_{j}
$$

where $p_{j}$ is a probability of a sample with amplitude $j, G$ is the greatest amplitude of the signal. In Table 2, signalto-noise ratio (SNR dB) values for different fuzzification methods and window sizes are shown. In Table 3 , the entropy-based compression ratio (ECR) for different fuzzification methods and window sizes are shown. These results show that our one-dimensional algorithm has a high data compression when using $L$ or $Z$ fuzzification methods. The results also indicate that by using the $Z$ fuzzification we achieve a higher compression ratio with good SNR than those results with $L$ fuzzification.

In the second example, we apply our two-dimensional FMP algorithm to process block by block the test image. The fuzzy structuring functions are generated by multiplying two one-dimensional structuring functions derived from DLO

\begin{tabular}{|c|c|c|c|c|c|c|c|c|}
\hline \multicolumn{3}{|c|}{$L$} & \multicolumn{3}{|c|}{$Z$} & \multicolumn{3}{|c|}{$S$} \\
\hline 3 & 4 & 5 & 3 & 4 & 5 & 3 & 4 & 5 \\
\hline 10.329 & 0.246 & 0.197 & 0.316 & 0.236 & 0.188 & 0.329 & 0.246 & 0.197 \\
\hline 20.492 & 0.362 & 0.286 & 0.356 & 0.271 & 0.220 & 0.542 & 0.389 & 0.303 \\
\hline 30.555 & 0.425 & 0.338 & 0.365 & 0.281 & 0.232 & 0.636 & 0.483 & 0.333 \\
\hline 4 & 0.470 & 0.355 & & 0.287 & 0.237 & & 0.498 & 0.397 \\
\hline 5 & & 0.379 & & & 0.241 & & & 0.428 \\
\hline
\end{tabular}

TABLE 3: Compression ratio for one-dimensional FMP.

\begin{tabular}{|c|c|c|c|c|c|c|c|c|c|}
\hline & \multicolumn{3}{|c|}{$L$} & \multicolumn{3}{|c|}{$Z$} & \multicolumn{3}{|c|}{$S$} \\
\hline & $3 \times 3$ & $4 \times 4$ & $5 \times 5$ & $3 \times 3$ & $4 \times 4$ & $5 \times 5$ & $3 \times 3$ & $4 \times 4$ & $5 \times 5$ \\
\hline 1 & 22.41 & 20.29 & 18.73 & 22.41 & 20.29 & 18.73 & 22.41 & 20.29 & 18.73 \\
\hline 2 & 24.54 & 21.89 & 20.69 & 24.56 & 21.91 & 20.66 & 24.49 & 21.84 & 20.67 \\
\hline 3 & 27.80 & 24.15 & 21.89 & 27.75 & 24.13 & 21.86 & 27.68 & 24.01 & 21.82 \\
\hline & : & : & . & : & : & : & : & : & : \\
\hline & 29.01 & 26.12 & 23.05 & 28.89 & 25.04 & 22.66 & 28.79 & 25.91 & 22.57 \\
\hline
\end{tabular}

TABLE 4: Signal to noise ratio $(\mathrm{dB})$ for two-dimensional FMP.

TABLE 5: Compression ratio for two-dimensional FMP.

\begin{tabular}{cccccccccc}
\hline & \multicolumn{1}{c}{$L$} & \multicolumn{9}{c}{$Z$} & \multicolumn{3}{c}{$S$} \\
window sizes \\
& \multicolumn{1}{c}{ winc } \\
& $3 \times 3$ & $4 \times 4$ & $5 \times 5$ & $3 \times 3$ & $4 \times 4$ & $5 \times 5$ & $3 \times 3$ & $4 \times 4$ & $5 \times 5$ \\
\hline 1 & 0.109 & 0.061 & 0.039 & 0.104 & 0.058 & 0.037 & 0.109 & 0.061 & 0.039 \\
2 & 0.160 & 0.088 & 0.057 & 0.115 & 0.065 & 0.043 & 0.178 & 0.095 & 0.061 \\
3 & 0.199 & 0.109 & 0.069 & 0.123 & 0.071 & 0.046 & 0.230 & 0.123 & 0.076 \\
$\vdots$ & $\vdots$ & $\vdots$ & $\vdots$ & $\vdots$ & $\vdots$ & $\vdots$ & $\vdots$ & $\vdots$ & $\vdots$ \\
All & 0.281 & 0.163 & 0.119 & 0.128 & 0.075 & 0.050 & 0.370 & 0.207 & 0.152 \\
\hline
\end{tabular}

polynomials. The order is determined by the structuring index method discussed before. In Table 4, we show the signal-to-noise ratio (SNR $\mathrm{dB}$ ) for different fuzzification methods and window sizes. In Table 5, the entropy-based compression ratio (ECR) for different fuzzification methods and window sizes is shown. Those results indicate that our two-dimensional algorithm has a higher performance when using $L$ and $Z$ fuzzification methods. The results also indicate that the $Z$ fuzzification achieves a higher compression ratio with good SNR than the $L$ fuzzification. To illustrate the results, we show in Figures 7(a) and 7(c) and Figures 7(b) and 7(d) the FMP component and the corresponding error images, when using window size of $1 \times 3$ and $3 \times 3$, for $L$ function fuzzification.

Since there is no published papers, as we know, using the fuzzy morphology approach for data compression, we want to show the high performance of our algorithm by comparing with those obtained by commonly used high performance method such as discrete cosine transform (DCT) [23]. In order to have a fair comparison, we use the coefficients with high energy of the DCT and FMP such that the "peak-to-peak" SNR of the reconstructed 


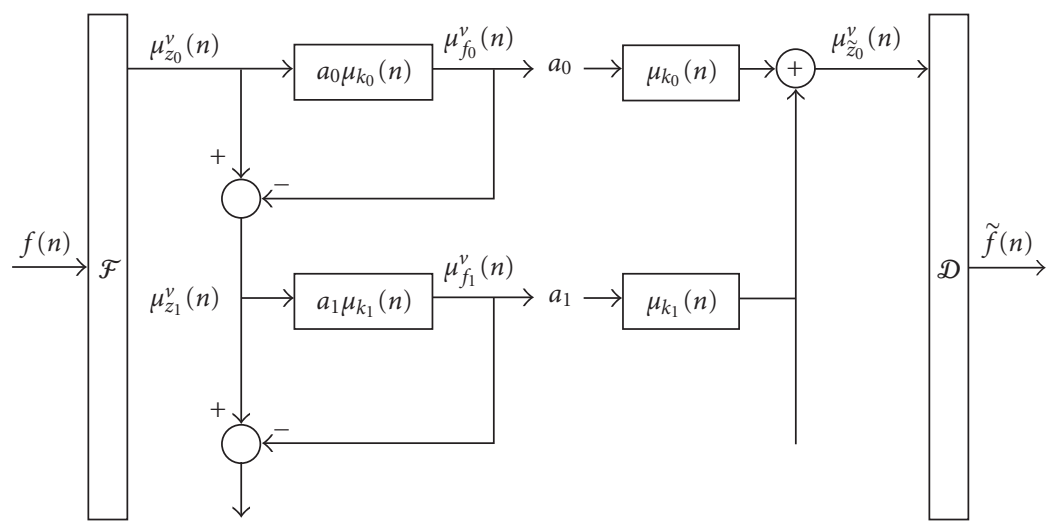

FIgURE 5: FMP representation block diagram.

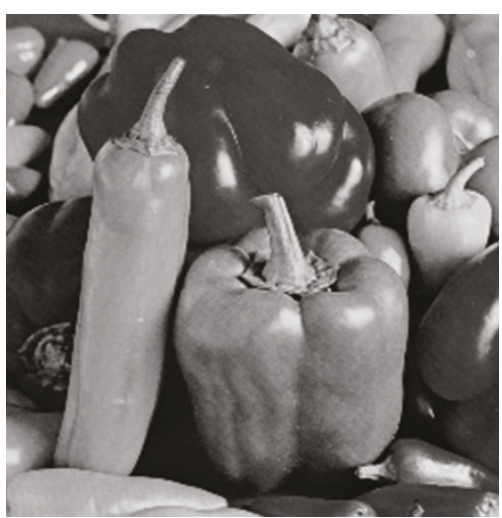

Figure 6: Original Image for analysis.

TABLE 6: Comparison for one-dimensional FMP and DCT.

\begin{tabular}{lcccccc}
\hline & \multicolumn{3}{c}{ FMP Z } & \multicolumn{3}{c}{ DCT } \\
& 3 & 4 & 5 & 3 & 4 & 5 \\
\hline SNR (dB) & 32.46 & 29.67 & 27.56 & 32.31 & 29.54 & 27.40 \\
ECR & 0.356 & 0.287 & 0.241 & 0.415 & 0.312 & 0.262 \\
\hline
\end{tabular}

image which is closely used in $[26,27]$, then we compare their data compression ratio at each different window size. The comparisons of SNR (dB) and ECR are shown in Tables 6 and 7 for one and two dimension, respectively. Those results indicate that our FMP representation using $Z$ fuzzification achieves the higher data compression ratio that of DCT. We also provide, as an example, the one- and twodimensional reconstructed images in Figure 8 using the FMP representation with $Z$ fuzzification and the DCT method, with window size of 3 and $3 \times 3$ cases, for visual quality comparison purpose.

Figures $8(\mathrm{a})$ and $8(\mathrm{c})$ and Figures $8(\mathrm{~b})$ and $8(\mathrm{~d})$ show the reconstructed images for one and two dimensions, respectively. Figures $8(\mathrm{a})$ and $8(\mathrm{~b})$ show the reconstructed images using FMP with $Z$ fuzzification. Figures $8(\mathrm{c})$ and 8 (d) show the the reconstructed images using DCT. Those
TABLE 7: Comparison for two-dimensional FMP and DCT.

\begin{tabular}{lcccccc}
\hline & \multicolumn{3}{c}{ FMP Z } & & DCT \\
& $3 \times 3$ & $4 \times 4$ & $5 \times 5$ & $3 \times 3$ & $4 \times 4$ & $5 \times 5$ \\
\hline SNR (dB) & 28.89 & 25.04 & 22.66 & 28.70 & 24.89 & 22.54 \\
ECR & 0.128 & 0.075 & 0.050 & 0.147 & 0.092 & 0.061 \\
\hline
\end{tabular}

TABLE 8: Computation Complexity of FMP and DCT.

\begin{tabular}{lcc}
\hline Operation & FMP & DCT \\
\hline Mul./Div. & $N^{2}$ & $3 N^{2}+3 N$ \\
Add./Sub. & $4 N$ & $2 N^{2}-N$ \\
Min./Max. & $N^{2}+2 N$ & - \\
Lut/Chk & $N^{2}-N$ & $N^{2}$ \\
Total & $3 N^{2}+5 N$ & $6 N^{2}+2 N$ \\
\hline
\end{tabular}

results indicate that the visual quality of using our FMP representation with $Z$ fuzzification and DCT method is similar. However, our FMP representation with $Z$ fuzzification achieves higher data compression ratio (fewer bits/pixel). For computation complexity comparison, Table 8 shows the required operations including addition/subtraction, multiplication/division, and minimum/maximum and look-up table/check-up for FMP and DCT. Assume a window size of $N$ is used. Notice that the multiplication/division and addition/subtraction operations contribute more computation complexity than the other operations. We can see clearly that the FMP requires less number of operations than that of the DCT.

5.2. Fractal Dimension Estimation Using FMP. To estimate the fractal dimension (FD) of one- or two-dimensional signals, we obtain the FMP representation of signal frames of increasing dimension. This representation is used to find an approximate cover of the windowed signal. It will be shown that when using the discrete Legendre polynomials as structuring functions, the covers can be obtained recursively providing a better FD estimation at each recursion. Our procedure can be easily extended to two dimensions. 


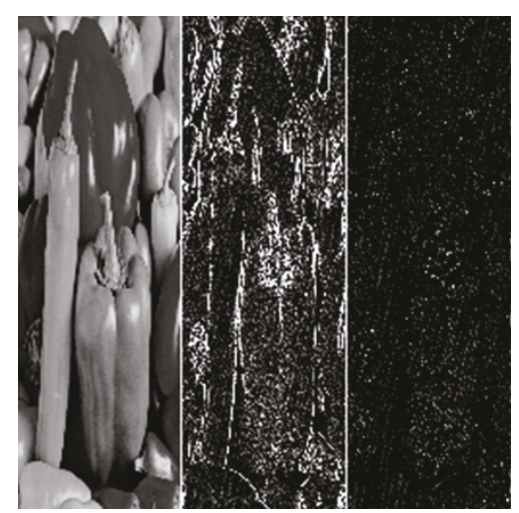

(a)

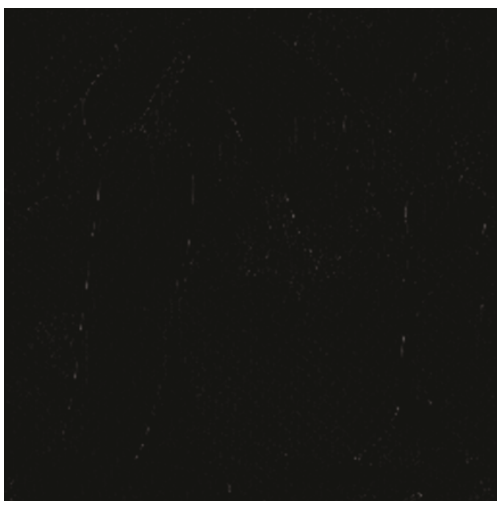

(c)

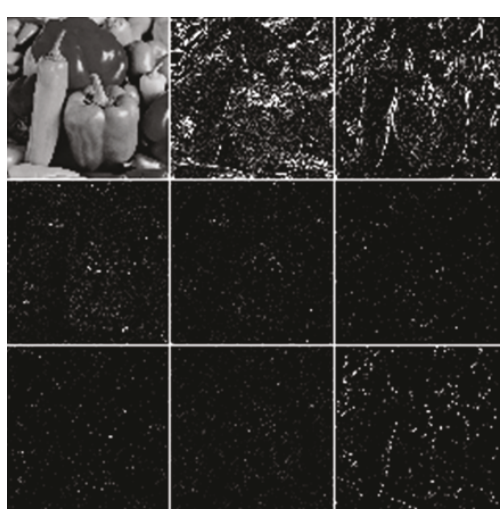

(b)

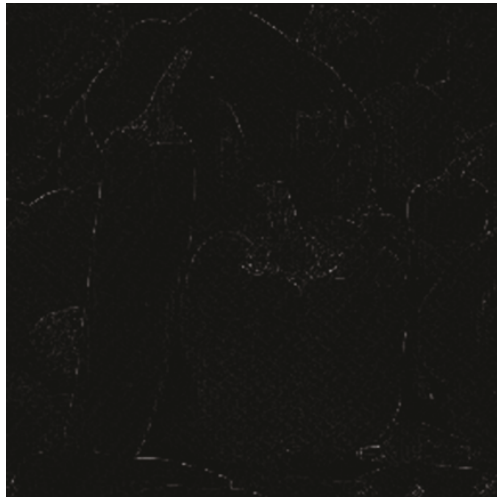

(d)

FIGURE 7: One- and two-dimensional FMP representation: (a) and (c) one-dimensional FMP component and error images, respectively, (b) and (d) two-dimensional FMP components and error image, respectively.

5.2.1. One-Dimensional Estimation. Let $\mu_{f}(n), 0 \leq n \leq$ $M-1$, be the membership function of a signal $f(n)$. For each of the signal frames, we will use $\left\{\mu_{k_{i}}(n)\right\}, 0 \leq n \leq$ $[0, N-1]$, as structuring functions based on the discrete Legendre polynomials (see Figure 3 when $N=5$ ). The FMP algorithm provides the adaptation parameters $\left\{a_{i}\right\}$, for each frame membership function $\mu_{z}(n)$. Let then the support length of $\mu_{f}(n)$ be $S=M-1$ (if we know the sampling period $T_{s}$ then $\left.S=(M-1) T_{s}\right)$ which is divided into an integer number of windows of increasing length $r=N-1$ (or $r=(N-1) T_{s}$ when $T_{s}$ is known).

For each of the frames, we will attempt to come up with a cover that encloses the signal as tightly as possible. The length of the cover can be calculated recursively from the FMP adaptive coefficients and the values of the structuring functions. For a frame $v$ with corresponding length $r$, we have that (See Figure 9 when $r=4$ )

$$
\begin{gathered}
\ell_{0}^{v}(r)=\frac{r}{S}, \\
\ell_{i}^{v}(r)=\sum_{j=1}^{r} \sqrt{\left(\mu_{k_{i}}(j)-\mu_{k_{i}}(j-1)\right)^{2}\left(a_{i}^{v}\right)^{2}+\left(\frac{\ell_{i-1}^{v}(r)}{r}\right)^{2}},
\end{gathered}
$$

where $\ell_{0}^{v}(r)$ is the geometric length corresponding to the constant FMP decomposition, and $i=1,2, \ldots, I, I \leq$ $r$ corresponds to the $i$ th FMP geometric decomposition. $j=1,2, \ldots, r$ corresponds to the point of the structuring function. The length calculation is done in each of the $r$ segments in which the window is divided (See Figure 9). The height of the the cover $d^{v}(r) \in[0,1]$ can be found exactly in the case where $\mu_{z}^{v}(n)$ is either a constant or has a great deal of variation. In the first case, $d^{v}(r)=0$, and in the second, $d^{v}(r)=1$. However, it should be noted that in these two cases, we will have $a_{i}^{v}=0, i>0$ that is only a constant approximation is possible. In cases different from the above ones, we cannot calculate $d^{v}(r)$ exactly although a good estimate of it can be obtained as the difference between the maximum and minimum of the residual $\mu_{z_{I+1}}^{v}(n)$ (see (18)) obtained after the $I$ th decomposition. Thus, the diagonal of the cover in frame $v$

$$
\ell^{v}(r)=\sqrt{\left(\ell_{I}^{v}(r)\right)^{2}+\left(d^{v}(r)\right)^{2}}
$$

can be used as an estimate of the length of the signal for that corresponding $r$.

Summing up the number of instruments contributions for each $r$,

$$
Q(r)=\sum_{v=1}^{[S / r]} \frac{\ell^{v}(r)}{\ell_{0}^{v}(r)}
$$




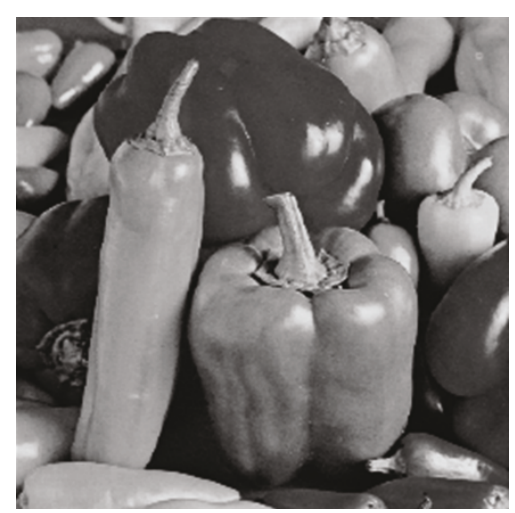

(a)

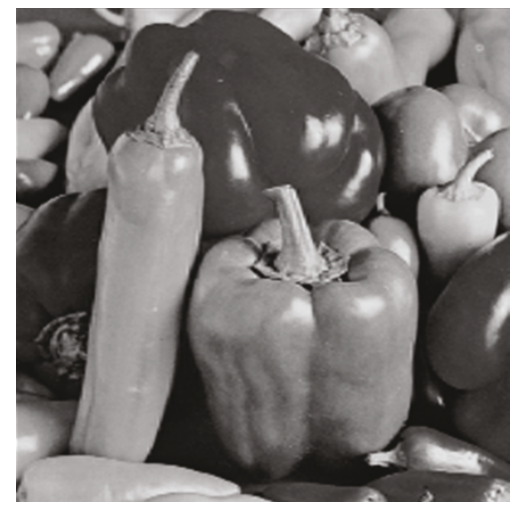

(c)

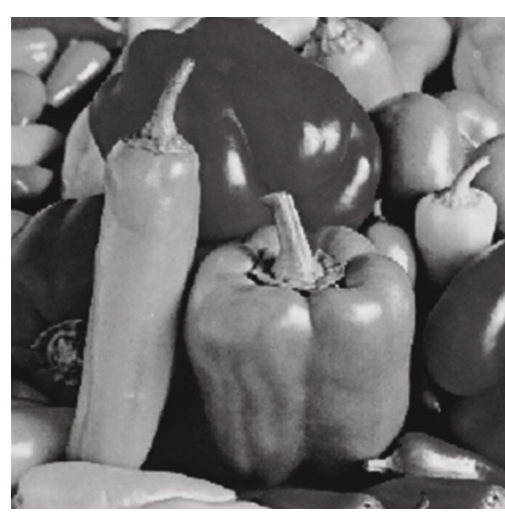

(b)

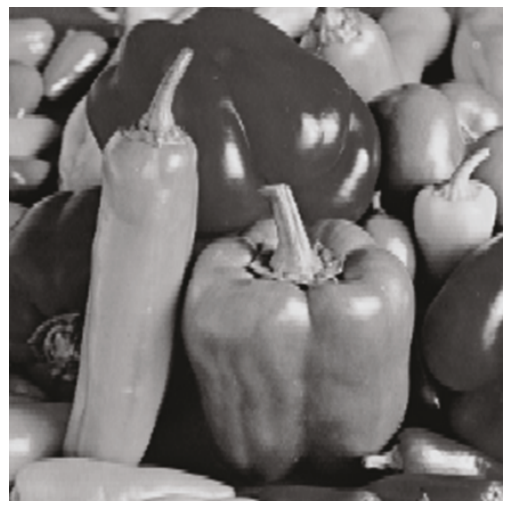

(d)

FIGURE 8: Reconstruction images: (a) and (c) for one-dimensional FMP $Z$ fuzzification and DCT method, respectively, (b) and (d) for two-dimensional FMP $Z$ fuzzification and DCT method, respectively.

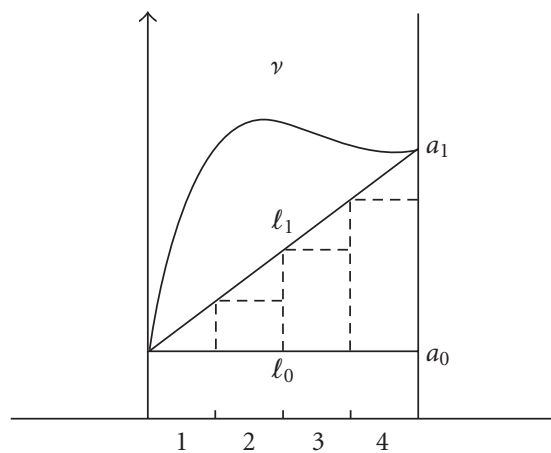

FIGURE 9: Frame signal length approximation.

Using least-square fitting in the $\log (Q(r))$ versus $\log (1 / r)$ graph for various values of $r$, the slope of the line will correspond to an estimate of the FD.

Remarks. (1) In order for the above discrete algorithm to work properly, we need to choose $r$ so that $S / r$ is an integer for every chosen value of $r$. Results vary for different choices of $r$ due to the global linear fitting. A better estimate might be obtained by doing the the linear fitting piecewise, obtaining a better estimate for linear fitting for small $r s$.

(2) If $\mu_{f}(n)$ is so smooth that the FMP gives $a_{i}^{v}=0$, $0<i \leq I$, for every $v$, then we have that $d^{v}(r) \approx 0$, and $\ell^{v}(r) / \ell_{0}^{v}(r)=1$ and, therefore, $Q(r)=S / r$ for any value of $r$. The estimation of the slope of $\log (Q(r))$ versus $\log (1 / r)$ for two different values of $r$ gives that the fractal dimension $D$ is one.

(3) On the other hand, if $\mu_{f}(n)$ varies widely everywhere, then the FMP gives $a_{i}^{v}=0,0 \leq i \leq I$, for every $v$, but due to the large variation $d^{v}(r) \approx 1$, in which case $\ell_{I}^{v}(r)=\ell_{0}^{v}(r)$. We will then get that

$$
\frac{\ell^{v}(r)}{\ell_{0}^{v}(r)}=\sqrt{1+\frac{S^{2}}{r^{2}}} \approx \frac{S}{r},
$$

after substituting $d^{v}(r)$ and $\ell_{0}^{v}(r)$, and using the fact that $S / r \gg 1$ (i.e., we divide $S$ into several windows) which will give us $Q(r)=S^{2} / r^{2}$, so that the slope estimation for two different values will give a fractal dimension close to two.

(4) According to 2 and 3 above mentioned we have that, except for very smooth and very rough signal, the FD will be a value between 1 and 2 . 


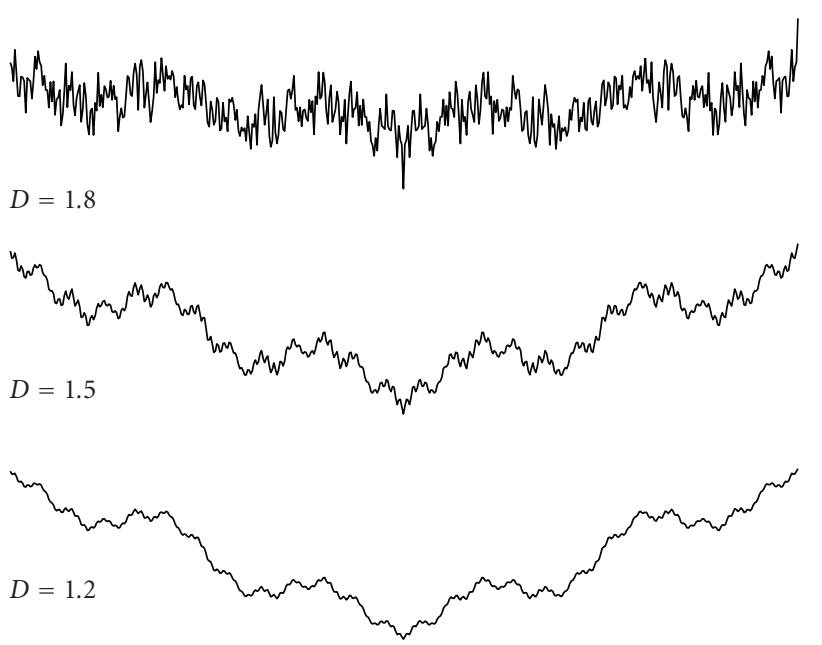

FIgURE 10: WCF generated signals with different FD.

TABLE 9: Estimated FD of WCF signal.

\begin{tabular}{cccccccc}
\hline True D & 1.2 & 1.3 & 1.4 & 1.5 & 1.6 & 1.7 & 1.8 \\
\hline Our D & 1.237 & 1.271 & 1.358 & 1.505 & 1.648 & 1.721 & 1.825 \\
MC D & 1.227 & 1.327 & 1.424 & 1.515 & 1.606 & 1.701 & 1.797 \\
\hline
\end{tabular}

(5) Due to the significance of the constant of the FMP approximation, a very efficient algorithm can be found when only that component is considered. We then have that

$$
\begin{aligned}
\ell^{v}(r) & =\sqrt{\left(\ell_{0}^{v}(r)\right)^{2}+\left(d^{v}(r)\right)^{2}}, \\
Q(r) & =\sum_{v=1}^{[S / r]} \sqrt{1+\left(\frac{d^{v}(r)}{\ell_{0}^{v}(r)}\right)^{2}},
\end{aligned}
$$

from which estimation of the FD can be done as before.

To test our algorithm, artificial fractal signals with known FD are generated using the weistrass cosine function (WCF) method [24, 28]. Figure 10 shows some WCF generated signals, and when we apply our procedure to them. The results are shown in Table 9. For comparison purpose, the results of using MC method [24] are also shown in the table.

5.2.2. Two-Dimensional Estimation. Different from the onedimensional case, the estimation of the FD of a twodimensional signal can only be done using the constant and linear term of the FMP approximation. Using just the first three terms of the approximation, we have for an $r \times r$ block of a square image of dimension $S \times S$ that get the following recursive formulas:

$$
\begin{gathered}
A_{0}^{u, v}(r)=\left(\frac{r}{S}\right)^{2}, \\
A_{i}^{u, v}(r)=\sqrt{\left(a_{i}^{u, v}\right)^{2} A_{i-1}^{u, v}(r)+\left(A_{i-1}^{u, v}(r)\right)^{2}},
\end{gathered}
$$

where $\left\{a_{i}^{u, v}\right\}$ are coefficients found using the FMP. Notice that these equations are similar to those in the onedimensional case, except that in this case the block is not subdivided as we did with the window in the onedimensional case. A cube covering the signal can be thought of having a base with area $A_{I}^{u, v}(r)$ and an approximate height of $d^{u, v}(r)$ equal to the difference between the maximum and the minimum of $\mu_{z}^{u, v}(n)$. The diagonal plane of this cube has an area equal to

$$
A^{u, v}(r)=\sqrt{\left(A_{I}^{u, v}(r)\right)^{2}+A_{I}^{u, v}(r)\left(d^{u, v}(r)\right)^{2}},
$$

a formulation analogous to that in the one-dimensional case, when we use only two components

$$
Q(r)=\sum_{u, v} \frac{A^{u, v}(r)}{A_{0}^{u, v}} .
$$

Similar comments as those made in the one-dimensional case can be made here. As before, $S / r$ must be an integer and the FD varies for different choices of $r$. Likewise a very smooth signal has an FD close to 2, and a very rough signal has an FD close to 3 .

Finally if in the FMP approximation we only use the $a_{0}$ component, the above algorithm simplifies to

$$
\begin{gathered}
A_{0}^{u, v}(r)=\left(\frac{r}{S}\right)^{2}, \\
A^{u, v}(r)=\sqrt{\left(A_{0}^{u, v}(r)\right)^{2}+A_{0}^{u, v}(r)\left(d^{u, v}(r)\right)^{2}}, \\
Q(r)=\sum_{u, v} \sqrt{1+\frac{\left(d^{u, v}(r)\right)^{2}}{A_{0}^{u, v}}}
\end{gathered}
$$

from which we can find an estimate of the FD of the given two-dimensional signal.

We then apply our algorithm to estimate the FD of the Brodatz texture images [29] shown in Figure 11. The code of texture image is same as in the Brodatz album. The results are shown in Table 10. This example shows the applicability of our algorithm to estimate FD to images. We also show the results of using DBC method [25] for comparison purpose. The results of using these two methods are similar.

\section{Conclusion}

A novel signal representation using fuzzy morphological approach has been proposed in this paper. Using the fuzzy morphological operators and a set of structuring functions, a decomposition and reconstruction procedure, similar to polynomial transform $[5,6]$, is developed for the fuzzified signals. Through using the fuzzy morphological approach, a signal can be efficiently represented with several additional advantages, such as lower computation complexity and flexible in using the different fuzzification methods to extract signal geometrical information for a better signal representation. Furthermore, our representation can be implemented very fast by parallel. We successfully use the fuzzy mathematical morphology [7] approach to extend the work 


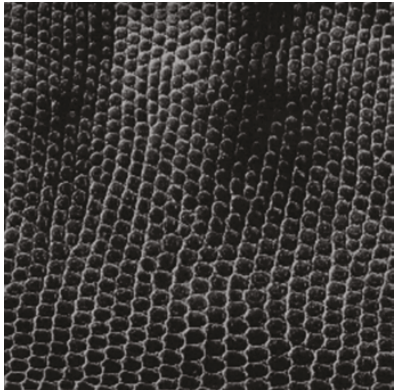

D03

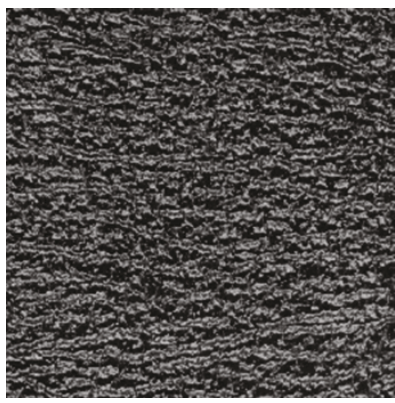

D24

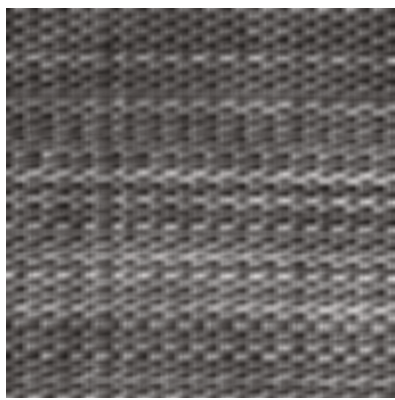

D25

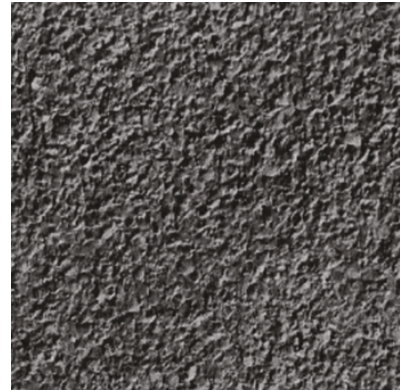

D04

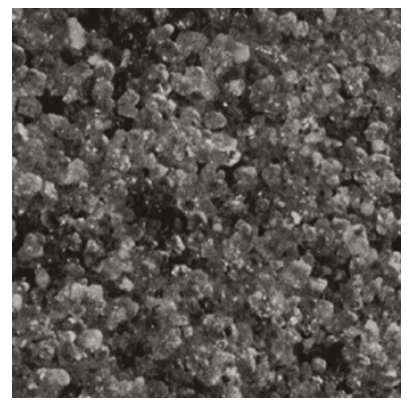

D28

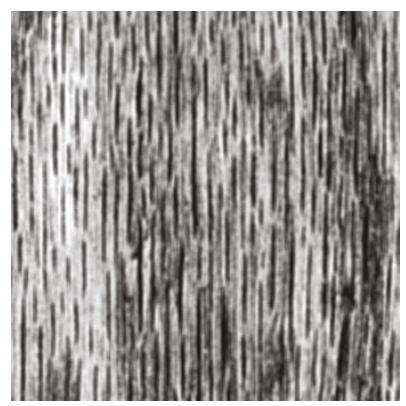

D68

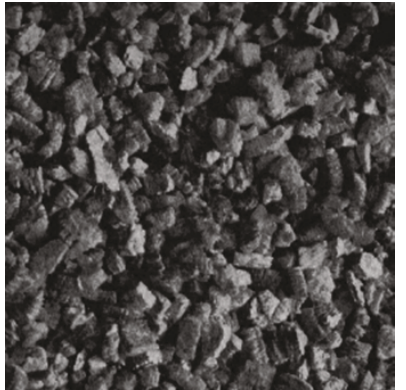

D05

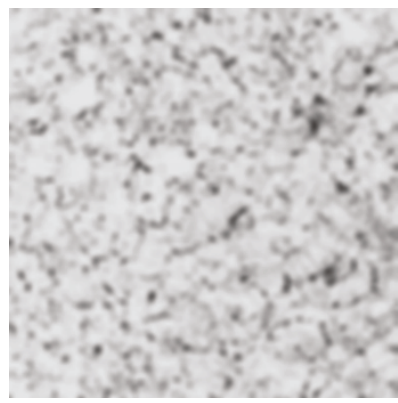

D33

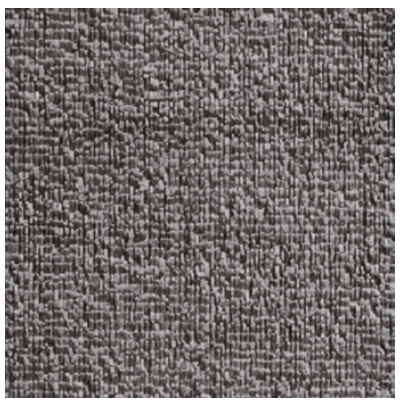

D84

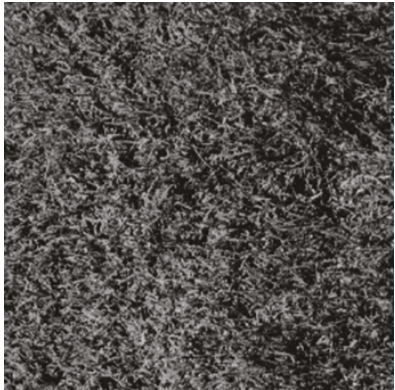

D09

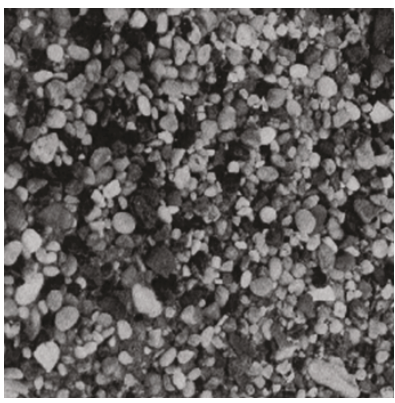

D54

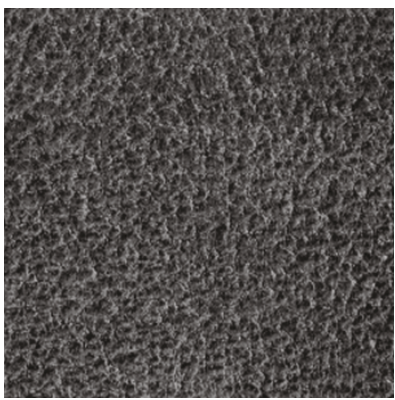

D92

Figure 11: Texture images.

TABLE 10: Estimated FD of texture images.

\begin{tabular}{|c|c|c|c|c|c|c|c|c|c|c|c|c|}
\hline & D03 & D04 & D05 & D09 & D24 & D28 & D33 & D54 & D55 & D68 & D84 & D92 \\
\hline Our D & 2.55 & 2.69 & 2.49 & 2.67 & 2.54 & 2.64 & 2.51 & 2.46 & 2.60 & 2.7 & 2.72 & 2.64 \\
\hline $\mathrm{DBC} \mathrm{D}$ & 2.60 & 2.66 & 2.54 & 2.59 & 2.54 & 2.55 & 2.23 & 2.39 & 2.48 & 2.52 & 2.60 & 2.50 \\
\hline
\end{tabular}

of the Pitas and Venetsanopoulos [8-10] and of Song and Delp [11] on morphological signal representation. We have applied our representation to data compression and fractal dimension estimation for one- and two-dimensional signals, the experimental results have shown the high performance in data compression and applicability in estimating fractal dimension as compared with those using the DCT [23], MC [24], and DBC [25] methods.

\section{Appendix}

Proof of Proposition 1. By definition

$$
\mu_{z_{i} \circ a_{i} k_{i}}=\mu_{\left(z_{i} \Theta a_{i} k_{i}\right) \oplus a_{i} k_{i}} .
$$

According to fuzzy morphological erosion

$$
\begin{aligned}
\mu_{z_{i} \Theta a_{i} k_{i}}(n) & =\min _{\ell, n+\ell}\left\{\min \left[1,1-a_{i} \mu_{k_{i}}(\ell)+\mu_{z_{i}}(n+\ell)\right]\right\} \\
& =\min _{\ell}\left\{\min \left[1,1-a_{i} \mu_{k_{i}}(\ell)+\mu_{z_{i}}(\ell)\right]\right\} \\
& =\mu_{c} \delta(n) .
\end{aligned}
$$

Now

$$
\begin{aligned}
& \begin{aligned}
\mu_{c \oplus a_{i} k_{i}}(n) & =\max _{\ell, n-\ell}\left\{\max \left[0, a_{i} \mu_{k_{i}}(\ell)+\mu_{c} \delta(n-\ell)-1\right]\right\} \\
& =\max \left[0, a_{i} \mu_{k_{i}}(n)+\mu_{c}-1\right] .
\end{aligned} \\
& \text { Thus, } \mu_{z_{i} \circ a_{i} k_{i}}(n)=\max \left[0, a_{i} \mu_{k_{i}}(n)+\mu_{c}-1\right] .
\end{aligned}
$$


Proof of Proposition 2. From Proposition 1,

$$
\mu_{z_{i} \circ a_{i} k_{i}}(n)= \begin{cases}a_{i} \mu_{k_{i}}(n)+\mu_{c}-1, & a_{i} \mu_{k_{i}}(n)+\mu_{c}-1 \geq 0, \\ 0, & a_{i} \mu_{k_{i}}(n)+\mu_{c}-1<0,\end{cases}
$$

One want $\mu_{z_{i} \circ a_{i} k_{i}}(n)=a_{i} \mu_{k_{i}}(n)$. Since $a_{i} \mu_{k_{i}}(n) \geq 0$, for all $n$, then a sufficient condition for this to happen is to set $\mu_{c}=1$. From Proposition 1, one can have $\mu_{c}=1+$ $\min \left\{0, \min _{\ell}\left[\mu_{z_{i}}(\ell)-a_{i} \mu_{k_{i}}(\ell)\right]\right\}$. That $\mu_{c}=1$ means that $\min \left\{0, \min _{\ell}\left[\mu_{z_{i}}(\ell)-a_{i} \mu_{k_{i}}(\ell)\right]\right\}=0$, which implies that

$$
\min _{\ell}\left[\mu_{z_{i}}(\ell)-a_{i} \mu_{k_{i}}(\ell)\right] \geq 0 \text {. }
$$

The optimum (maximum) value of $a_{i}$ (denoted as $a_{i}^{*}$ ) satisfying (A.5) is reached when the equality holds.

(1) Existence of $a_{i}^{*}$.

By contradiction, assume for all $a_{i} \in[0,1]$ such that $\min _{\ell}\left[\mu_{z_{i}}(\ell)-a_{i} \mu_{k_{i}}(\ell)\right] \neq 0$. There are two cases:

(a) $\min _{\ell}\left[\mu_{z_{i}}(\ell)-a_{i} \mu_{k_{i}}(\ell)\right]>0 . \Rightarrow \mu_{z_{i}}(\ell)>$ $a_{i} \mu_{k_{i}}(\ell)$ for all $\ell$, which is not true, in particular when $a_{i}=1$ and $\mu_{k_{i}}(\ell)=1$ for some $\ell$.

(b) when $\min _{\ell}\left[\mu_{z_{i}}(\ell)-a_{i} \mu_{k_{i}}(\ell)\right]<0$ is similarly shown.

(2) Uniqueness of $a_{i}^{*}$.

$$
\begin{aligned}
& \min _{\ell}\left[\mu_{z_{i}}(\ell)-a_{i} \mu_{k_{i}}(\ell)\right]=0 \Rightarrow \\
& \mu_{z_{i}}(\ell) \geq a_{i} \mu_{k_{i}}(\ell), \quad \forall \ell .
\end{aligned}
$$

If $\mu_{k_{i}}(\ell)=0$, for some $\ell \in[0, N-1]$, then (A.6) is always satisfied, so one needs to consider only when $\mu_{k_{i}}(\ell)>0$, for which $a_{i} \leq \mu_{z_{i}}(\ell) / \mu_{k_{i}}(\ell)$, Therefore,

$$
a_{i}^{*}=\min _{\substack{0 \leq \ell \leq N-1 \\ \mu_{k_{i}}(\ell) \neq 0}}\left\{\frac{\mu_{z_{i}}(\ell)}{\mu_{k_{i}}(\ell)}\right\} \text {. }
$$

Proof of Corollary 1. (i) It follows from Proposition 2. If $a_{i}>$ $a_{i}^{*}$, then

$$
\mu_{z_{i}}(\ell)-a_{i} \mu_{k_{i}}(\ell)<\mu_{z_{i}}(\ell)-a_{i}^{*} \mu_{k_{i}}(\ell)
$$

taking minimum over $\ell$, we get $J\left(a_{i}\right)<J\left(a_{i}^{*}\right)=0$.

(ii) If $a_{i}<a_{i}^{*}$ can be similarly shown.

(iii) By Proposition 2 and $\mu_{k_{0}}(n)=1$.

(iv) By the antiextensive property of opening and optimum condition $\mu_{z_{i} \circ a_{i}^{*} k_{i}}(n)=a_{i}^{*} \mu_{k_{i}}(n) \leq \mu_{z_{i}}(n)$.

By Proposition $1 \mu_{z_{i} \circ a_{i}^{*} k_{i}}(n) \geq 0 \Rightarrow 0 \leq a_{i}^{*} \mu_{k_{i}}(n) \leq$ $\mu_{z_{i}}(n)$. Thus, $0 \leq a_{i}^{*} \max _{n}\left[\mu_{k_{i}}(n)\right] \leq \max _{n}\left[\mu_{z_{i}}(n)\right] \leq 1$, and $0 \leq a_{i}^{*} \leq \max _{n}\left[\mu_{z_{i}}(n)\right] \leq 1$.

Proof of Corollary 2. By Proposition 2 and $\mu_{k_{0}}(n)=1 . a_{0}^{*}=$ $\min _{n}\left[\mu_{z_{0}^{c}}(n)\right]$. Thus, $a_{0}^{* c}=\max _{n}\left[\mu_{z_{0}}(n)\right]$.
Proof of Proposition 3. (i) According to the antiextensive property of opening $\mu_{z_{i} \circ a_{i} k_{i}}(n) \leq \mu_{z_{i}}(n)$ then $\mu_{z_{i+1}}(n)=$ $\mu_{z_{i}}(n)-\mu_{z_{i} \circ a_{i} k_{i}}(n) \geq 0$, so $\mu_{z_{i+1}}(n) \leq \mu_{z_{i}}(n)$.

(ii) By (17) and Proposition 2, we get

$$
\min _{n}\left[\mu_{z_{i+1}}(n)\right]=\min _{n}\left[\mu_{z_{i}}(n)-\mu_{z_{i} \circ a_{i} k_{i}}(n)\right]=0 .
$$

Proof of Proposition 4. This can be proven easily using (22) and (23).

Proof of Proposition 5. By definition,

$$
\mu_{k_{i, j}}(m, n)=\mu_{k_{i}}(m) \mu_{k_{j}}(n) .
$$

We have

$$
\begin{aligned}
\mu_{k_{0, j}}(m, n) & =\mu_{k_{0}}(m) \mu_{k_{j}}(n) \\
& \geq \mu_{k_{i}}(m) \mu_{k_{j}}(n), \quad \forall m, n, i, j .
\end{aligned}
$$

Notice that $\mu_{k_{0}}(\ell) \geq \mu_{k_{i}}(\ell)$, for all $i, \ell$.

(ii) and (iii) are similarly shown.

Proof of Proposition 6. By optimum condition,

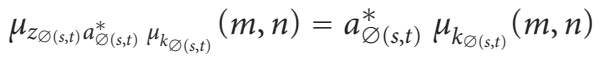

$$
\begin{aligned}
& a_{\phi(s, t)}^{*} \\
& =\min _{\substack{p, q \\
\mu_{k_{\phi(s, t)}}(p, q) \neq 0}}\left\{\frac{\mu_{z_{\phi(s, t)}}(p, q)}{\mu_{k_{\phi(s, t)}}(p, q)}\right\} \geq 0 \\
& =\min _{\substack{p, q \\
\mu_{k_{\phi(s, t)}}(p, q) \neq 0}}\left\{\frac{\mu_{z_{\phi(i, j)}}(p, q)-a_{\phi(i, j)}^{*} \mu_{k_{\phi(i, j)}}(p, q)-\in(p, q)}{\mu_{k_{\phi(s, t)}}(p, q)}\right\} \\
& \leq \min _{\substack{p, q \\
\mu_{k_{\phi(s, t)}}(p, q) \neq 0}}\left\{\frac{\mu_{z_{\phi(i, j)}}(p, q)-a_{\phi(i, j)}^{*} \mu_{k_{\phi(i, j)}}(p, q)}{\mu_{k_{\phi(s, t)}}(p, q)}\right\} \\
& \leq \min _{\substack{p, q \\
\mu_{k_{\phi(i, j)}}(p, q) \neq 0}}\left\{\frac{\mu_{z_{\phi(i, j)}}(p, q)-a_{\phi(i, j)}^{*} \mu_{k_{\phi(i, j)}}(p, q)}{\mu_{k_{\phi(i, j)}}(p, q)}\right\} \\
& =a_{\phi(i, j)}^{*}-a_{\phi(i, j)}^{*} \\
& =0 \text {. }
\end{aligned}
$$

Therefore, $a_{\phi(s, t)}^{*}=0$.

\section{References}

[1] J.-L. Starck, M. Elad, and D. L. Donoho, "Image decomposition via the combination of sparse representations and a variational approach," IEEE Transactions on Image Processing, vol. 14, no. 10, pp. 1570-1582, 2005.

[2] M. Unser and D. Van De Ville, "The pairing of a wavelet basis with a mildly redundant analysis via subband regression," IEEE Transactions on Image Processing, vol. 17, no. 11, pp. 2040-2052, 2008. 
[3] S. G. Mallat, "Theory for multiresolution signal decomposition: the wavelet representation," IEEE Transactions on Pattern Analysis and Machine Intelligence, vol. 11, no. 7, pp. 674-693, 1989.

[4] S. G. Mallat, "Multifrequency channel decompositions of images and wavelet models," IEEE Transactions on Acoustics, Speech, and Signal Processing, vol. 37, no. 12, pp. 2091-2110, 1989.

[5] J. Martens, "The Hermite transform-theory," IEEE Transactions on Acoustics, Speech, and Signal Processing, vol. 38, no. 9, pp. 1595-1606, 1990.

[6] J. Martens, "The Hermite transform-applications," IEEE Transactions on Acoustics, Speech, and Signal Processing, vol. 38, no. 9, pp. 1607-1618, 1990.

[7] D. Shinha and E. R. Dougherty, "Fuzzy mathematical morphology," Journel of Visual Communication and Image Processing, pp. 286-302, 1992.

[8] I. Pitas and A. N. Venetsanopoulos, "Morphological shape decomposition," IEEE Transactions on Pattern Analysis and Machine Intelligence, vol. 12, no. 1, pp. 38-45, 1990.

[9] I. Pitas, "Morphological signal decomposition," in Proceedings of the International Conference on Acoustics, Speech, and Signal Processing (ASSP '90), pp. 2169-2172, Albuquerque, NM, USA, April 1990.

[10] I. Pitas and A. N. Venetsanopoulos, "Morphological shape representation," in Proceedings of the International Conference on Acoustics, Speech, and Signal Processing (ICASSP '91), pp. 2381-2384, Toronto, Canada, May 1991.

[11] J. Song and E. J. Delp, "The analysis of morphological filters with multiple structuring elements," Computer Vision, Graphics and Image Processing, vol. 50, no. 3, pp. 308-328, 1990.

[12] C. Huang and L. F. Chaparro, "Signal representation using fuzzy morphology," in Proceedings of the 3rd International Symposium on Uncertainty Modeling and Analysis and Annual Conference of the North American Fuzzy Information Processing Society, (ISUMA-NAFIPS '95), pp. 607-612, IEEE, September 1995.

[13] G. Matheron, Random Sets and Integral Geometry, Wiely, New York, NY, USA, 1973.

[14] L. A. Zadeh, "Fuzzy sets," Information and Control, vol. 8, no. 3, pp. 338-353, 1965.

[15] D. Dubois and H. Prade, Fuzzy Sets and Systems Theory and Applications, Academic Press, New York, NY, USA, 1980.

[16] H. Bandemer and W. Nather, Fuzzy Data Analysis, Kluwer Academic Publishers, Dordrecht, The Netherlands, 1992.

[17] L. A. Zadeh, K. S. Fu, K. Tanaka, and M. Shimura, Fuzzy Sets and Their Applications to Cognitive and Decision Processes, Academic Press, New York, NY, USA, 1975.

[18] J. Serra, Image Analysis and Mathematical Morphology, Academic Press, New York, NY, USA, 1982.

[19] A. N. Evans and X. U. Liu, "A morphological gradient approach to color edge detection," IEEE Transactions on Image Processing, vol. 15, no. 6, pp. 1454-1463, 2006.

[20] H. Cha and L. F. Chaparro, "A morphological polynomial transform," in Proceedings of the International Conference on Acoustics, Speech, and Signal Processing (ICASSP '93), vol. 5, pp. 173-176, April 1993.

[21] C. P. Neuman and D. I. Schonbach, "Discrete (legendre) orthogonal polynomials-a survey," International Journal for Numerical Methods in Engineering, vol. 8, pp. 743-770, 1974.
[22] L. F. Chaparro and M. Boudaoud, "Two-dimensional linear prediction covariance method and its recursive solution," IEEE Transactions on Systems, Man and Cybernetics, vol. 17, no. 4, pp. 617-621, 1987.

[23] R. Gonzales and R. E. Woods, Digital Image Processing, Prentice-Hall, Englewood Cliffs, NJ, USA, 3rd edition, 2008.

[24] P. Maragos and F. Sun, "Measuring the fractal dimension of signals: morphological covers and iterative optimization," IEEE Transactions on Signal Processing, vol. 41, no. 1, pp. 108121, 1993.

[25] N. Sarkar and B. B. Chauduri, "Efficient differential boxcounting approach to compute fractal dimension of image," IEEE Transactions on Systems, Man and Cybernetics, vol. 24, no. 1, pp. 115-120, 1994.

[26] S.-H. Jung, S. K. Mitra, and D. Mukherjee, "Subband DCT: definition, analysis, and applications," IEEE Transactions on Circuits and Systems for Video Technology, vol. 6, no. 3, pp. 273-286, 1996.

[27] B. Kosko, Neural Network and Fuzzy System, Prentice-Hall, Englewood Cliffs, NJ, USA, 1991.

[28] B. B. Mandelbrot, The Fractal Geometry of Nature, W. H. Freeman, San Francisco, Calif, USA, 1982.

[29] P. Brodatz, Textures, Dover, New York, NY, USA, 1966. 